\title{
The Immediate Effect of Neuromuscular Joint Facilitation (NJF) Treatment for Knee Osteoarthritis
}

\author{
KOUJi NAKATA, OT ${ }^{1)}$, Misato KOUGO, $\mathrm{PT}^{1)}$, \\ Ming HuO, PT, $\mathrm{PhD}^{2)}$, Hitoshi MARUYAMA, PT, $\mathrm{PhD}^{2)}$ \\ 1) Department of Rehabilitation, Umimura Branch Hospital \\ 2) Department of Physical Therapy, Faculty of Health Science, International University of Health and \\ Welfare: 2600-1 Kitakanemaru, Ohtawara City, Tochigi 324-8501, Japan. \\ TEL+81287-24-3000,E-mail: huoming8@gmail.com
}

\begin{abstract}
Purpose] The aim of this study was to investigate the changes of pain severity and walking ability of elderly people with knee osteoarthritis (OA) after neuromuscular joint facilitation (NJF) treatment. [Subjects] The subjects were twenty elderly people with knee OA ( 7 males, 13 females), and the subjects were divided into two groups: a NJF group and a control group. [Methods] Participants in the NJF group received NJF treatment. Participants in the control group received the conventional rehabilitation treatment. The changes in pain severity were assessed with a visual analogue scale (VAS), and the changes in walking ability were assessed with walking velocity, step length, and cadence. [Results] The NJF group showed significant increases in walking velocity, step length and decreased cadence. VAS was significantly decreased in both groups. [Conclusion] Our results show that NJF treatment not only decreased pain severity, but also improved the walking ability of elderly subjects with knee osteoarthritis.
\end{abstract}

Key words: Knee osteoarthritis, Neuromuscular joint facilitation, Tibia rotation function

(This article was submitted Jul. 27, 2011, and was accepted Sep. 7, 2011)

\section{INTRODUCTION}

Osteoarthritis (OA), one of the most common joint diseases in adults ${ }^{1,2)}$, is a slowly evolving and degenerative articular disease. Among elderly people's knee joint diseases, OA is the most general diseases, and $25-40 \%$ of middle-aged and elderly persons suffer from this disease regardless of the presence of symptoms. The pathophysiologies of knee OA are degeneration and wear loss of the articular cartilage. The incidence of primary OA without anamnestic history such as traumas and infectious disorders is $90 \%{ }^{3)}$. The primary complaints of patients are pain, stiffness, instability and loss of function.

Neuromuscular Joint Facilitation (NJF) is a new therapeutic exercise based on kinesiology, and integrates the facilitation element of proprioceptive neuromusclar facilitation and the joint composition movement, aiming to improve the movement of the joint through passive exercise, active exercise and resistance exercise ${ }^{4)}$. It is used to increase strength, flexibility and ROM.

The aim of this study was to investigate the changes of pain severity and walking ability in elderly people with knee OA before and after NJF treatment.

\section{SUBJECTS AND METHODS}

The subjects were twenty elderly people with knee OA (7 males, 13 females) who attended our hospital as outpatients.
They were assigned randomly to two groups: a NJF group ( 3 males, 7 females) and a control group ( 4 males, 6 females). Subject characteristics are detailed in Table 1. The patients had clinical symptoms and signs consistent with knee OA, and met the grade II criteria suggested by Altman $^{5)}$. All subjects were screened before the start of the study by filling out a medical history questionnaire. The questionnaire addressed whether the subjects had a history of cardiopulmonary, somatosensory or neurological disorders, or severe visual and vestibular loss. If so, they were excluded from the study. All subjects gave their informed consent to participate in the study.

An occupational therapist conducted the clinical examination, which included an assessment of pain severity using the visual analogue scale (VAS), and measurements of the $5 \mathrm{~m}$ maximum walking tome and number of steps; the walking velocity, step length and cadence were calculated.

Table 1. Subject Characteristics ${ }^{\mathrm{a}}$

\begin{tabular}{lccc}
\hline & $\begin{array}{c}\text { NJF }^{\mathrm{a}} \\
(\mathrm{n}=10)\end{array}$ & $\begin{array}{c}\text { Control }^{\mathrm{b}} \\
(\mathrm{n}=10)\end{array}$ & $\begin{array}{c}\text { Sum total } \\
(\mathrm{n}=20)\end{array}$ \\
\hline Age $(\mathrm{y})$ & $74.0 \pm 6.0$ & $76.7 \pm 6.6$ & $75.4 \pm 6.3$ \\
Height $(\mathrm{cm})$ & $159.5 \pm 7.1$ & $154.7 \pm 9.8$ & $157.1 \pm 8.7$ \\
Weight $(\mathrm{kg})$ & $58.2 \pm 7.7$ & $54.8 \pm 11.5$ & $56.5 \pm 9.7$ \\
\hline
\end{tabular}

Note: values are mean \pm standard deviation. There were no significant differences between the groups. ${ }^{\text {aa }}$ NJF group: neuromuscular joint facilitation group. ${ }^{\text {b: }}$ Control: control group. 
Knee pain was determined using a VAS. A $10 \mathrm{~cm}$ line was drawn, and the left end was labeled "no pain" and the right end was labeled "the worst pain experienced". Each subject was asked to mark the level of pain before and after treatment, and the distance from the left end to the mark was measured.

To measure the $5 \mathrm{~m}$ maximum walking velocity, lines were drawn at the $1 \mathrm{~m}$ and $6 \mathrm{~m}$ points on a straight walking track of $7 \mathrm{~m}$, and the time taken walk between the $1 \mathrm{~m}$ and $6 \mathrm{~m}$ points was recorded as the walking time as well as the number of steps. The $5 \mathrm{~m}$ maximum walking time was measured 2 times, and the mean value was used in the analysis.

Four knee patterns of NJF were used. The patterns were the knee extension-tibia external rotation (E-ER) pattern, the knee flexion-tibia internal rotation (F-IR) pattern, the knee extension- tibia internal rotation (E-IR) pattern, and the knee flexion- tibia external rotation (F-ER) pattern. Each pattern was performed three times at random as a passive exercise and a resistance exercise. To measure the resistance force of the resistance exercise, two hand-held dynamometers (HHD, ANIMA MT-1) were held in each hand of a physical therapist and the maximal resistance force was measured using the tester function of HHD. Table 2 shows the resistance force and duration of the procedure. The control group performed conventional therapeutic exercises.

To determine differences between the NJF group and the control group, the independent t-test was performed on subject characteristics. The Mann-Whitney test was performed to investigate the differences between groups. The Wilcoxon test was performed to investigate the differences between before and after intervention. Data were analyzed using SPSS Ver. 12.0 for Windows. The level of statistical significance was chosen as 0.05 .

\section{RESULTS}

Table 3 shows the results for each measurement. The walking velocity and the step length significantly increased, and the cadence significantly decreased in post- intervention measurements as compared to pre-intervention measure-
Table 2. The resistance force and duration of NJF treatment for the knee joint $(\mathrm{n}=6)$

\begin{tabular}{lccc}
\hline Pattern & $\begin{array}{c}\text { Proximal resistance } \\
\text { force }(\mathrm{kg})\end{array}$ & $\begin{array}{c}\text { Distal resistance } \\
\text { force }(\mathrm{kg})\end{array}$ & $\begin{array}{c}\text { Duration of } \\
\text { procedure }(\mathrm{sec})\end{array}$ \\
\hline E-ER $^{\mathrm{a}}$ & $11.6 \pm 1.9$ & $14.3 \pm 2.1$ & $3.8 \pm 0.6$ \\
F-IR $^{\mathrm{b}}$ & $12.2 \pm 1.5$ & $11.2 \pm 1.9$ & $4.1 \pm 0.3$ \\
E-IR $^{\mathrm{c}}$ & $14.4 \pm 2.1$ & $9.9 \pm 0.8$ & $3.5 \pm 0.6$ \\
F-ER $^{\mathrm{d}}$ & $8.9 \pm 1.1$ & $12.2 \pm 1.8$ & $4.2 \pm 0.4$ \\
\hline
\end{tabular}

a: E-ER: knee extension-tibia external rotation pattern. ${ }^{\text {b: }}$ F-IR: knee flexion-tibia internal rotation pattern. ${ }^{c}$ : E-IR: knee extension- tibia internal rotation pattern. ${ }^{d}$ : F-ER: knee flexion- tibia external rotation pattern.

ments in the NJF group. VAS was significantly lower after the intervention in both groups.

In the post-intervention between-group comparison the NJF group had a significantly lower VAS and a significantly longer step length than the control group.

\section{DISCUSSION}

This study investigated the immediate effects of a Neuromuscular Joint Facilitation treatment on pain and the walking ability of elderly people with knee osteoarthritis. The NJF group subjects showed not only decreased pain severity, but also improved walking ability.

An important difference between the intervention methods was that, the rotation function of the tibia was emphasized in the NJF group. In the NJF resistance exercise, the tibia rotation was drawn out using proximal resistance and traction. The results suggest that tibia rotation function can be improved in elderly people with knee OA by NJF treatment, and that improvement of tibia rotation contributes to walking ability. The walking velocity and the step length were increased after the NJF intervention, and the cadence was decreased, demonstrating that subject gait was improved by NJF treatment.

Future studies are needed to investigate the changes in pain severity and walking ability after a long period of NJF treatment.

Table3. Comparison of before and after intervention of each measurement item

\begin{tabular}{llcc}
\hline & & NJF $^{\mathrm{a}}(\mathrm{n}=10)$ & Control $^{\mathrm{b}}(\mathrm{n}=10)$ \\
\hline \multirow{2}{*}{$\operatorname{VAS}^{\mathrm{c}}(\mathrm{mm})$} & before & $22.6 \pm 15.8$ & $30.1 \pm 15.9$ \\
& after & $0.0 \pm 0.0 \dagger$ & $12.0 \pm 12.2 \ddagger^{* *}$ \\
Walking velocity (m/min) & before & $62.5 \pm 14.9$ & $52.3 \pm 23.8$ \\
& after & $68.0 \pm 15.4 \ddagger$ & $50.0 \pm 22.4$ \\
Step length (m) & before & $0.53 \pm 0.10$ & $0.47 \pm 0.16$ \\
& after & $0.64 \pm 0.13 \ddagger$ & $0.47 \pm 0.16^{*}$ \\
Cadence (steps/min) & before & $117.6 \pm 12.0$ & $106.8 \pm 21.6$ \\
& after & $106.2 \pm 10.2 \ddagger$ & $103.2 \pm 21.6$ \\
\hline
\end{tabular}

Note: values are mean \pm standard deviation. Comparison between groups: MannWhitney test. $*: \mathrm{p}<0.05 ; * *: \mathrm{p}<0.01$. Comparison before and after intervention: Wilcoxon test. $\uparrow: p<0.05 ; \ddagger: p<0.01$. ${ }^{a}$ : NJF group: neuromuscular joint facilitation group. ${ }^{\text {b: }}$ Control: control group. ${ }^{c}$ : VAS:Visual analogue scale to evaluate the pain level of the knee joint. 


\section{REFERENCES}

1) Anderson J, Felson DT: Factors associated with osteoarthritis of the knee in the first National Health and Nutrition Examination survey. Am J Epidemiol, 1988, 128: 179-189.

2) Sharma C, Dougherty DD, Felson D: The prevalence of patellofemoral and mixed compartment involvement in knee osteoarthritis and their effect on functional status. J Invest Med, 1996, 44: 359A.

3) Koshino $T$ : The manual of knee medical examination. Tokyo: Ishiyaku press, $1995, \mathrm{p} 1$.

4) Huo M: Neuromuscular Joint Facilitation. Tokyo: Ipec press, 2010, p 3.

5) Altman R, Asch E, Bloch D, et al.: Development of criteria for the classification and reporting of osteoarthritis. Classification of osteoarthritis of the knee. Arthritis Rheumatology, 1986, 29: 1039-1049. 